\title{
Overexpression of PFKFB3 Promotes Cell Glycolysis and Proliferation in Renal Cell Carcinoma
}

Jun Li

The Seventh Affiliated Hospital Sun Yat-sen University https://orcid.org/0000-0003-1227-6939

\section{Shiqiang Zhang}

The Seventh Affiliated Hospital Sun Yat-sen University

\section{Dingzhun Liao}

The Seventh Affiliated Hospital Sun Yat-sen University

\section{Qian Zhang}

The Seventh Affiliated Hospital Sun Yat-sen University

\section{Chujie Chen}

The Seventh Affiliated Hospital Sun Yat-sen University

\section{Xiangwei Yang}

The Seventh Affiliated Hospital Sun Yat-sen University

\section{Donggen Jiang}

The Seventh Affiliated Hospital Sun Yat-sen University

Jun Pang ( $\sim$ pangjun2@mail.sysu.edu.cn )

The Seventh Affiliated Hospital, Sun Yat-sen University https://orcid.org/0000-0003-0024-9415

\section{Research}

Keywords: Renal cell carcinoma, PFKFB3, Glycolysis, Proliferation, G1/S transition

Posted Date: March 2nd, 2021

DOI: https://doi.org/10.21203/rs.3.rs-255023/v1

License: (c) (1) This work is licensed under a Creative Commons Attribution 4.0 International License. Read Full License

Version of Record: A version of this preprint was published at BMC Cancer on January 20th, 2022. See the published version at https://doi.org/10.1186/s12885-022-09183-2. 


\section{Abstract}

Background: Cancer cells prefer aerobic glycolysis to increase their biomass and sustain uncontrolled proliferation. As a key glycolytic activator, phosphofructo-2-kinase/fructose-2,6-bisphosphatase 3 (PFKFB3) has been implicated in the progression of multiple types of tumors. However, the specific function and clinical significance of PFKFB3 in renal cell carcinoma (RCC) remain unclear. In the present study, we explored the role of PFKFB3 in RCC.

Methods: We analyzed the expression of PFKFB3 in clear cell renal cell carcinoma (ccRCC) tissues and its relationship with clinical characteristics of ccRCC. Real-time PCR and Western blot analysis were used to detect PFKFB3 expression levels in different RCC cell lines. Furthermore, we determined the glycolytic activity by glucose uptake, lactate secretion assay and ECAR analysis. CCK-8 assay, clone formation assay, flow cytometry and EdU assay were performed to monitor cancer cell proliferation and cell cycle distribution. In addition, nude mice xenograft model was used to investigate the role of PFKFB3 in tumor growth in vivo.

Results: In this study, we found that PFKFB3 was significantly up-regulated in RCC tissues and cell lines compared with normal control. Overexpression of PFKFB3 was positively associated with advanced TNM stage and could predict poor prognosis of ccRCC patients. Furthermore, knockdown of PFKFB3 suppresses cell glycolysis, proliferation and cell cycle G1/S transition in RCC cells. Importantly, in vivo experiments confirmed that PFKFB3 knockdown delayed tumor growth derived from the ACHN cell line.

Conclusion: Our results suggest that PFKFB3 plays an important role in the progression of RCC via mediating glycolysis and proliferation, and provides a potential therapeutic target for RCC.

\section{Introduction}

The incidence of renal cell carcinoma (RCC) has been increasing, with more than 400,000 estimated new cases and more than 170,000 deaths globally in 2018 [1]. As the most common type of RCC, clear cell renal cell carcinoma (ccRCC) accounts for the vast majority (approximately 70-75\%) [2]. Early-stage nonmetastatic RCC can be treated by radical or partial nephrectomy, however, many patients present with advanced disease that has a much poorer prognosis at the time of diagnosis because early-stage disease is often asymptomatic [3]. Targeted therapy against vascular endothelial growth factor (VEGF) and novel immunotherapy agents has been approved and used for the treatment of advanced or metastatic RCC. However, the therapeutic effects are limited for a short time and the patients eventually relapses [4]. Hence, it is essential to better understand the molecular mechanism of the oncogenesis and progression of RCC to provide guidance for early diagnosis and novel therapeutic strategies. Recently, new therapeutic strategies have emerged as effective treatment options against advanced RCC through the reprogramming of cancer cell metabolism and use of glycolysis inhibitor [5].

Rapid proliferation and glucose metabolism remodeling are hallmarks of cancer. To provide sufficient energy and support rapid biosynthesis, cancer cells exhibit enhanced glycolysis, even in the presence of 
normal oxygen supply. This phenomenon is historically known as the Warburg effect [6-7]. Exploring the cooperative mechanisms underlying cell proliferation and glycolysis could contribute to a better understanding of human cancer development. During glycolysis, Phosphofructo-2-kinase/fructose-2,6biophosphatase 3 (PFKFB3) catalyzes the synthesis of fructose-2,6-bisphosphate (F-2,6-BP), a potent allosteric stimulator of the key enzyme 6-phosphofructo-1-kinase 1 (PFK-1) [8]. Thus, the activation of PFKFB3 has been linked to enhanced glycolysis. PFKFB3 has been found to be overexpressed in various types of tumor, such as pancreatic cancer [9], breast cancer [10], and gastric cancer [11]. Upregulated PFKFB3 levels have been proved to be associated with poorer survival statistics in patients with breast cancer [12]. PFKFB3 plays a pivotal role in promoting cell proliferation by regulating the expression of important cell cycle proteins: cyclin-dependent kinase-1 (CDK1) and p27 [13]. Inhibition of PFKFB3 by genetic silence or chemical inhibitors obviously reduces glycolytic flux, Ras-driven transformation and tumor growth in nude mice [14-15]. Taken together, these results suggest that PFKFB3 plays important roles in several biological processes and in the progression of human cancers. However, the oncogenic role of PFKFB3 in RCC has been little explored.

In the present study, we detected the expression of PFKFB3 in $90 \mathrm{ccRCC}$ patients and analyzed the correlation of PFKFB3 level with clinicopathological features and prognosis. Furthermore, we investigated whether PFKFB3 knockdown inhibits glycolytic activity and cell proliferation in vitro and in vivo.

\section{Methods And Materials}

\section{Patients and tissue samples}

The tissue microarray (TMA) including 90 pairs of ccRCC tissues and matched adjacent tissues was purchased from Shanghai Outdo Biotech Company. All patients were pathologically diagnosed with ccRCC and received no additional treatment before surgery. Patients were further divided into subgroups using the TNM stage classification (stage I, II, III and IV) and Fuhrman grade system (Grade I, II, III and IV). This study was conducted in compliance with the Declaration of Helsinki and approved by the Ethics Committee of the Affiliated Hospital of Xuzhou Medical University. Informed consent was obtained from all subjects.

\section{Immunohistochemistry (IHC)}

IHC assays were performed as previously described [16]. Anti-PFKFB3 (1:250, Abcam, ab181861), and anti-Ki67 antibodies (1:250, Abcam, ab15580) were used. Because PFKFB3 protein is primarily located in the nucleus, we applied $\mathrm{H}$-score to assess the immunoreactivity of PFKFB3 according to the staining intensity and percentage. Staining intensity in cells was scored 0 3 (negative; weak; moderate; intense) and this value was multiplied by the percentage $(0 \% \sim 100 \%)$ of cells staining positively to generate an $\mathrm{H}$ score (range, 0 300). Each slide was independently assessed and averaged by two pathologists without prior knowledge of patient data. 


\section{Cell culture}

The human RCC cell lines were obtained from the American Type Culture Collection (ATCC). Cells were cultured in RPMI-1640 (HyClone) supplemented with 10\% fetal bovine serum (Invitrogen) and 1\% penicillin/streptomycin (HyClone) at $37^{\circ} \mathrm{C}$ with $5 \% \mathrm{CO}_{2}$.

\section{Lentiviral vector construction and transduction}

Lentiviral vectors carrying shRNA targeting PFKFB3 (sh PFKFB3) were designed and synthesized by GenePharma (Shanghai, China). Lentiviruses carrying shRNA targeting firefly luciferase (sh Ctrl) were used as the control. Cells were transduced with the lentiviruses and selected with puromycin as previously described [17]. Finally, the stable cell lines were verified using Western blot.

\section{RNA isolation and Real-time quantitative PCR}

RNA was extracted using TRIzol (Invitrogen) and cDNA was synthesized using the RevertAid First Strand CDNA Synthesis kit (Takara, Japan). Real-time PCR was carried out on ABI-7500 using SYBR ${ }^{\circledR}$ Premix Ex $\mathrm{Taq}^{\text {TM }}$ (Takara, Japan). $\beta$-actin was used as the internal control gene. The primers used in this study were as follows: PFKFB3 forward 5'-GGCC GCATCGGGGGCGACTC-3' and reverse 5'-TTGCGTCTCAGCTC AGGGAC-3'; $\beta$-actin forward 5'-GGGACCTGACTGACTAC-3' and reverse 5'-TCATACTCCTGCTTGCTGAT-3'. The results were normalized to $\beta$-actin expression and the fold changes were calculated using the $2^{-\triangle \triangle C T}$ method.

\section{Western blot analysis}

Western blot analysis was performed as described previously [16]. Cells were lysed in RIPA buffer. The protein concentrations were normalized with a BCA assay kit (Thermo Fisher Scientific, USA). $\beta$-actin was used as the loading control. Anti-PFKFB3 (1:1000, Abcam, ab181861) and anti- $\beta$-actin antibodies (1:3000, Abcam, ab179467) were used in this study.

\section{Glucose uptake and lactate secretion analysis}

Glucose and lactate concentrations were measured using a Glucose Colorimetric Assay Kit (BioVision, USA) and a Lactate Assay Kit (BioVision, USA), respectively, according to the manufacturer's instructions. Statistical differences were calculated compared with the negative control group.

\section{Extracellular acidification rate (ECAR) analysis}


The glycolysis capacity was determined using a Seahorse XF Glycolysis Stress Test Kit (Agilent, Santa Clara, USA) according to the manufacturer's instructions. In brief, $4 \times 10^{4}$ cells were seeded in 96 -well plates and incubated overnight. After washing the cells with Seahorse buffer, $10 \mathrm{mmol} / \mathrm{L}$ glucose, 1 $\mathrm{mmol} / \mathrm{L}$ oligomycin, and $100 \mathrm{mmol} / \mathrm{L}$ 2-deoxy-glucose (25 $\mathrm{mL}$ of each) were added to measure the ECAR. ECAR values were calculated and normalized to the cell number.

\section{Cell proliferation and cell cycle analysis}

Cell proliferation was examined by Cell Counting Kit-8 (CCK-8) assay, colony formation assay, cell cycle analysis and 5-ethynyl-2-deoxyuridine (EdU) incorporation assay, as described previously [18]. Cell cycle analysis was conducted using FACS cytometry (Beckman Coulter, USA).

\section{Tumor xenograft growth in nude mice}

Animal experiments were conducted as described previously [19]. Four-week-old male BALB/c nude mice were obtained from Sun Yat-sen University Experimental Animal Center. A total of $5 \times 10^{6} \mathrm{ACHN}$ cells transduced with either sh Ctrl and sh PFKFB3 were injected subcutaneously into the dorsal thighs of mice. The tumor sizes were recorded regularly every week and the tumor volume $\left(\mathrm{mm}^{3}\right)$ was calculated as follows: Volume $=0.5 \times$ length $\times$ width ${ }^{2}$. After five weeks, mice were sacrificed by $\mathrm{CO}_{2}$ inhalation, then the tumor nodules were dissected, weighed and sectioned, followed by IHC analysis. This study was approved by the Experimental Animal Care Commission of Sun Yat-sen University. All procedures performed in this study were in accordance with the ethical standards of the institutional ethics committee and with the NC3Rs ARRIVE guidelines.

\section{Statistical analysis}

The data were presented as the mean $\pm S D$ from at least three independent experiments. Statistical analysis was performed using SPSS 16.0 software. The differences between groups were calculated using Student's $t$ test or one-way ANOVA. Survival analyses were performed using the Kaplan-Meier method and assessed using the log-rank test. All the statistical teste were two-sided, Pख0.05 were considered statistically significant.

\section{Results}

\section{PFKFB3 protein is overexpressed in human ccRCC specimens and positively correlates with advanced TNM stage and poor prognosis of cCRCC patients}


Using IHC, we detected the expression levels of PFKFB3 protein in ccRCC tissues and tumor-adjacent normal tissues. As shown in Fig.1a-b, we demonstrated that immunostaining signals of PFKFB3 were strong in the nucleus of ccRCC cells but weak in normal renal tissues. The H-score of the ccRCC tissues and tumor-adjacent normal tissues were $131.52 \pm 5.61$ and $72.36 \pm 4.55$, respectively, and the difference between the two tissue types was statistically significant (Fig.1C). By analyzing the relationship between the expression levels of PFKFB3 and pivotal clinicopathological features of ccRCC patients, we found

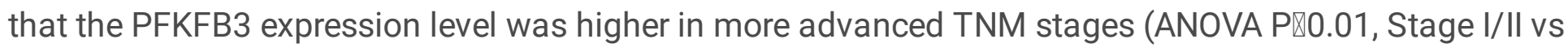
III/IV, $P \otimes 0.01$, Fig.1d), while increased expression of PFKFB3 in higher Fuhrman grades was observed, but not statistically significant (ANOVA $P=0.1156$, Grade I/II vs III/IV, $P=0.1302$, Fig.1e). Additionally, we explored the prognostic value of PFKFB3, patients were classified into two groups: low PFKFB3 expression group ( $\mathrm{H}$-score冈the median, $\mathrm{n}=41)$ and high PFKFB3 expression ( $\mathrm{H}$-score邓the median, $\mathrm{n}=41)$, eight patients were excluded because of loss of follow-up. Kaplan-Meier analysis showed that ccRCC patients with high PFKFB3 expression exhibited worse overall survival (Fig.1f). Moreover, multivariate Cox analysis indicated that PFKFB3 was an independent prognostic factor in ccRCC patients (Table 1).

Table 1

Correlation between the PFKFB3 level and the overall survival of ccRCC patients

\begin{tabular}{|c|c|c|c|}
\hline Clinical Variables & $\mathrm{HR}$ & $95 \% \mathrm{Cl}$ & P-value \\
\hline \multicolumn{4}{|l|}{ Univariate analysis } \\
\hline Age ( $\geq 60$ vs $₫ 60)$ & 1.875 & $1.152-3.149$ & 0.015 \\
\hline Gender (Male vs Female) & 1.267 & $0.589-2.627$ & 0.365 \\
\hline 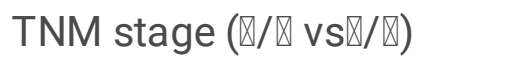 & 3.396 & $2.133-5.548$ & $₫ 0.001$ \\
\hline 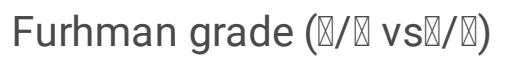 & 2.376 & $1.116-4.385$ & 0.003 \\
\hline PFKFB3 (High vs Low) & 1.715 & $1.232-2.540$ & $₫ 0.001$ \\
\hline \multicolumn{4}{|l|}{ Multivariate analysis } \\
\hline Age ( $\geq 60$ vs $₫ 60)$ & 1.394 & $0.925-2.214$ & 0.065 \\
\hline 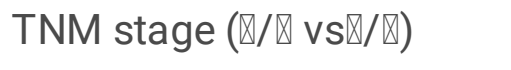 & 2.674 & $1.878-3.794$ & $₫ 0.001 *$ \\
\hline 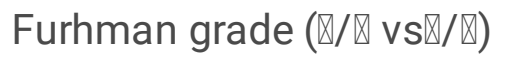 & 1.751 & $1.130-2.812$ & $0.009 *$ \\
\hline PFKFB3 (High vs Low) & 1.358 & $1.028-2.791$ & $\triangle 0.001 *$ \\
\hline
\end{tabular}

\section{PFKFB3 expression levels are higher in RCC cells}

To investigate the role of PFKFB3 in RCC progression, we first used qRT-PCR and Western blot analysis to detect the mRNA and protein levels of PFKFB3 in an immortalized normal human proximal tubule epithelial cell line (HK-2) and a panel of RCC cell lines (ACHN, Caki-2, A498, 786-0, OS-RC-1). As shown in 
Figure 2A, the mRNA expression levels of PFKFB3 were upregulated in the RCC cell lines compared with HK-2 cells. In addition, Western blot analysis results indicated that PFKFB3 protein expression levels were higher in RCC cells than in normal HK-2 cells, and the difference was statistically significant (Fig.2b-c).

\section{Knockdown of PFKFB3 inhibits glycolysis in RCC cells}

Considering PFKFB3 is a key protein that regulates cell glycolysis, we further explored the effects of PFKFB3 knockdown on glycolysis in RCC cells. In this study, we performed loss-of-function experiments by PFKFB3 shRNA. ACHN and A498 cells were selected because they had the highest PFKFB3 mRNA expression among the RCC cell lines (Fig.2a). The knockdown efficiency of PFKFB3 was confirmed by Western blot (Fig.3a-b). As shown in Fig.3c-d, knockdown of PFKFB3 in ACHN and A498 cells strikingly reduced the glucose intake and lactate secretion capacity. Moreover, the extracellular acidification rate (ECAR) of RCC cells was measured using a Seahorse XF24e Extracellular Flux Analyzer, we found that basal glycolysis, maximal glycolytic capacity and glycolytic reserve of $\mathrm{ACHN}$ and $\mathrm{A} 498$ cells were restrained after transfection of PFKFB3 shRNA (Fig.3e-g), which insinuated that glycolytic potency of RCC cells might be attenuated by PFKFB3 shRNA. Taken together, our data indicated that PFKFB3 is functionally important in aerobic glycolysis of RCC cells.

\section{Down-regulation of PFKFB3 suppresses proliferation and G1/S transition in RCC cells}

To further elucidate the functional role of PFKFB3 in cell proliferation, ACHN and A498 cells were transfected with the indicated PFKFB3 shRNA as mentioned before. The CCK-8 assay revealed that PFKFB3 knockdown obviously reduced the cell proliferation ability of ACHN and A498 cells (Fig.3a-b). Moreover, the colony formation assay confirmed that both ACHN and A498 cells formed fewer and smaller colonies after transfection of PFKFB3 shRNA (Fig.3c-d).

To dissect the biological events accompanying the alterations of cell proliferation caused by PFKFB3, and FACS was applied to analyze the distribution of cell cycle phases. As shown in Fig.4e-f, knockdown of PFKFB3 in ACHN and A498 cells displayed a significant increase in the percentage of cells in G1-phase but decreased proportions of S-phase cells. In addition, EdU incorporation assay demonstrated that ACHN and A498 cells contained less EdU-positive cells with newly synthesized DNA after transfection of PFKFB3 shRNA (Fig.4g-h). Therefore, these data suggested that PFKFB3 might accelerate the G1/S transition of cell-cycle progression and consequently promoted the proliferation of RCC cells.

\section{PFKFB3 knockdown inhibits RCC tumor growth in vivo}

To test the impact of PFKFB3 on the tumor growth in vivo, ACHN cells with PFKFB3 shRNA were injected into nude mice tumors subcutaneously. As expected, the tumors with PFKFB3 knockdown displayed a 
marked reduction in tumor volume and weight compared with the control group (Fig.5a-c). Furthermore, immunohistochemical staining confirmed that PFKFB3 knockdown decreased the levels of the cell proliferation marker Ki-67 (Fig.5d-e). These results are consistent with our in vitro experimental results. Taken together, our data indicated that downregulation of PFKFB3 effectively suppresses the RCC tumors in vivo.

\section{Discussion}

Cancer cells reprogram glucose metabolism toward aerobic glycolysis to increase their biomass and maintain uncontrolled proliferation. PFKFB3 acts as a major regulator of high glycolytic flux in cancers by catalyzing the synthesis of fructose 2,6-biphosphate, which activates 6-phosphofructo-1-kinase. Several recent studies have indicated that PFKFB3 was highly expressed in multiple human cancers [9-11], while there are few reports on the expression of PFKFB3 in RCC. In this study, we detected the expression of PFKFB3 in 100 ccRCC patients and found that the levels of PFKFB3 expression were upregulated in cCRCC tissues compared to tumor-adjacent normal tissues. Moreover, overexpression of PFKFB3 in CCRCC tissues was positively associated with tumor stage but independent of tumor grade, suggesting that PFKFB3 played critical roles in cancer cells by linking glycolysis to cell proliferation.

Most of the literature has reported that increasing PFKFB3 promotes tumorigenesis and proliferation [2022]. However, experiment result in astrocytoma cells is inconsistent. Zscharnack et al. [23] found that PFKFB3 splice variant UBI2K4 was downregulated in high-grade astrocytoma compared to low-grade astrocytoma and corresponding non-neoplastic brain tissue. Overexpression of UBI2K4 suppressed cell viability and anchorage-independent growth of U87 cells. Therefore, further study is needed to elaborate the exact role of PFKFB3 in different cancer cells. In the present study, endogenous PFKFB3 expression in all RCC cell lines tested was strikingly higher than that in the normal human proximal tubule epithelial cell line HK-2. Because of the very high basal level of PFKFB3 in all kinds of RCC cells, we performed loss-offunction experiments in vitro and in vivo by silencing PFKFB3 expression to investigate the biological effect of PFKFB3. Among these various RCC cell lines, the two with the highest mRNA expression of PFKFB3 were selected. Our loss-of-function studies demonstrated that PFKFB3 inhibition significantly decreased glycolysis and proliferation in RCC cells both in vitro and in vivo. Moreover, we found that PFKFB3 knockdown blocked the G1/S transition in RCC cells, suggesting that this inhibition may prevent cancer cell proliferation. A previous study reported that silencing of PFKFB3 inhibited glycolysis and induced G2 phase cell cycle arrest in HeLa cells [24]. Although the glycolytic role of PKFFB3 in cancer progression has been the subject of numerous functional studies, some scholars have also focused on the functions of PFKFB3 beyond glycolysis. For example, Yalcin et al. [13] found that PFKFB3 knockdown triggered cell cycle arrest in G1 phase and caused an elevated expression of p27 protein in HeLa cells, and the effect of siRNA silencing of PFKFB3 was reversed by co-siRNA silencing of $p 27$. It is well known that p27 is a potent suppressor of the G1/S transition and activator of apoptosis [25]. Other studies have also shown that PFKFB3 was transported to the nucleus in cancer cells and ectopic expression of wildtype PFKFB3 in the nucleus promoted cell proliferation without an effect on glycolysis [26]. These results suggested that the role of PFKFB3 in tumorigenesis was dependent on not only its function of regulating 
glucose metabolism, but also its function of controlling cell cycle. The detailed relationship between these phenomena and the specific molecular mechanism of PFKFB3 still needed to be further explored.

There were some limitations in our study. For example, we detected the expression levels of PFKFB3 in a panel of RCC cells lines, but no one with a low basal level of endogenous PFKFB3 was found. Therefore, gain-of-function analysis was not performed because the results could not reflect the biological function of PFKFB3 well in the RCC cells. Additionally, the exact molecular mechanism of PFKFB3 on the proliferation of RCC cells remains elusive. Further research on this issue may bring new ideas and strategies for the diagnosis and treatment of RCC.

\section{Conclusion}

In summary, we demonstrated that PFKFB3 is upregulated in RCC tissues and cell lines. Overexpression of PFKFB3 is positively associated with advanced TNM stage and poor prognosis of ccRCC patients. PFKFB3 knockdown suppresses glycolysis, proliferation, and blocked the G1/S transition in RCC cells. Our findings suggest that PFKFB3 plays an important role in the progression of RCC, and provides a potential therapeutic target for RCC.

\section{Declarations}

\section{Funding}

This study was supported by the National Nature Science Foundation of China (81772754), Major Basic Research and Cultivation Program of Natural Science Foundation of Guangdong Province (2017A03038009), the Medical Science and Technology Foundation of Guangdong Province (A2019555), Shenzhen Basic Science Research (JCYJ20190809164617205), Sanming Project of Shenzhen (SZSM202011011), and Research start-up fund of part-time PI, SAHSYSU (ZSQYJZPI202003).

\section{Conflicts of interest}

The authors declare that they have no potential conflicts of interest.

\section{Availability of data and materials}

The datasets used and/or analyzed during the current study are available from the corresponding author on reasonable request.

\section{Authors' contributions}


JP and JL designed the research. All authors contribute to data analysis, drafting or revising the article, gave final approval of the version to be published, and agree to be accountable for all aspects of the work.

\section{Compliance with ethical standards}

\section{Ethics approval and consent to participate}

The tissue microarray (TMA) analysis in this study was conducted in compliance with the Declaration of Helsinki and approved by the Ethics Committee of the Affiliated Hospital of Xuzhou Medical University. Informed consent was obtained from all subjects. Animal experiments have been approved by the ethics committee of Sun Yat-sen University.

\section{Consent for publication}

All authors agreed to publish the article.

\section{References}

1. Bray F, Ferlay J, Soerjomataram I, Siegel RL, Torre LA, Jemal A. Global cancer statistics 2018 : GLOBOCAN estimates of incidence and mortality worldwide for 36 cancers in 185 countries. CA Cancer J Clin. 2018;68(6):394-424.

2. Shuch B, Amin A, Armstrong AJ, Eble JN, Ficarra V, Lopez-Beltran A, Martignoni G, Rini BI, Kutikov A. Understanding pathologic variants of renal cell carcinoma: distilling therapeutic opportunities from biologic complexity. Eur Urol. 2015;67(1):85-97.

3. Escudier B, Porta C, Schmidinger M, Rioux-Leclercq N, Bex A, Khoo V, Grünwald V, Gillessen S, Horwich A; ESMO Guidelines Committee. Electronic address: clinicalguidelines@esmo.org. Renal cell carcinoma: ESMO Clinical Practice Guidelines for diagnosis, treatment and follow-upt. Ann Oncol. 2019;30(5):706-20.

4. Lalani AA, McGregor BA, Albiges L, Choueiri TK, Motzer R, Powles T, Wood C, Bex A. Systemic Treatment of Metastatic Clear Cell Renal Cell Carcinoma in 2018: Current Paradigms, Use of Immunotherapy, and Future Directions. Eur Urol. 2019;75(1):100-10.

5. Son JY, Yoon S, Tae IH, Park YJ, De U, Jeon Y, Park YJ, Rhyu IJ, Lee BM, Chung KH, Lim JE, Lee SJ, Lee HW, Kwak JH, Kim HS, Choi HY. Novel therapeutic roles of MC-4 in combination with everolimus against advanced renal cell carcinoma by dual targeting of Akt/pyruvate kinase muscle isozyme M2 and mechanistic target of rapamycin complex 1 pathways. Cancer Med. 2018;7(10):5083-95.

6. WARBURG O. On the origin of cancer cells. Science. 1956;123(3191):309-14.

7. WARBURG O. On respiratory impairment in cancer cells. Science. 1956;124(3215):269-70. 
8. Shen J, Jin Z, Lv H, Jin K, Jonas K, Zhu C, Chen B. PFKP is highly expressed in lung cancer and regulates glucose metabolism. Cell Oncol (Dordr). 2020;43(4):617-29.

9. Richardson DA, Sritangos P, James AD, Sultan A, Bruce JIE. Metabolic regulation of calcium pumps in pancreatic cancer: role of phosphofructokinase-fructose-bisphosphatase-3 (PFKFB3). Cancer Metab. 2020;8:2.

10. O'Neal J, Clem A, Reynolds L, Dougherty S, Imbert-Fernandez Y, Telang S, Chesney J, Clem BF. Inhibition of 6-phosphofructo-2-kinase (PFKFB3) suppresses glucose metabolism and the growth of HER2+ breast cancer. Breast Cancer Res Treat. 2016;160(1):29-40.

11. Han J, Meng Q, Xi Q, Wang H, Wu G. PFKFB3 was overexpressed in gastric cancer patients and promoted the proliferation and migration of gastric cancer cells. Cancer Biomark. 2017;18(3):249-56.

12. Peng F, Li Q, Sun JY, Luo Y, Chen M, Bao Y. PFKFB3 is involved in breast cancer proliferation, migration, invasion and angiogenesis. Int J Oncol. 2018;52(3):945-54.

13. Yalcin A, Clem BF, Imbert-Fernandez Y, Ozcan SC, Peker S, O'Neal J, Klarer AC, Clem AL, Telang S, Chesney J. 6-Phosphofructo-2-kinase (PFKFB3) promotes cell cycle progression and suppresses apoptosis via Cdk1-mediated phosphorylation of p27. Cell Death Dis. 2014;5(7):e1337.

14. Telang S, Yalcin A, Clem AL, Bucala R, Lane AN, Eaton JW, Chesney J. Ras transformation requires metabolic control by 6-phosphofructo-2-kinase. Oncogene. 2006;25(55):7225-34.

15. Clem B, Telang S, Clem A, Yalcin A, Meier J, Simmons A, Rasku MA, Arumugam S, Dean WL, Eaton J, Lane A, Trent JO, Chesney J. Small-molecule inhibition of 6-phosphofructo-2-kinase activity suppresses glycolytic flux and tumor growth. Mol Cancer Ther. 2008;7(1):110-20.

16. Li X, Li J, Cai Y, Peng S, Wang J, Xiao Z, Wang Y, Tao Y, Li J, Leng Q, Wu D, Yang S, Ji Z, Han Y, Li L, Gao X, Zeng C, Wen X. Hyperglycaemia-induced miR-301a promotes cell proliferation by repressing p21 and Smad4 in prostate cancer. Cancer Lett. 2018;418:211-20.

17. Ju HQ, Ying H, Tian T, Ling J, Fu J, Lu Y, Wu M, Yang L, Achreja A, Chen G, Zhuang Z, Wang H, Nagrath D, Yao J, Hung MC, DePinho RA, Huang P, Xu RH, Chiao PJ. Mutant Kras- and p16-regulated NOX4 activation overcomes metabolic checkpoints in development of pancreatic ductal adenocarcinoma. Nat Commun. 2017;8:14437.

18. Li J, Jiang D, Zhang Q, Peng S, Liao G, Yang X, Tang J, Xiong H, Pang J. MiR-301a Promotes Cell Proliferation by Repressing PTEN in Renal Cell Carcinoma. Cancer Manag Res. 2020;12:4309-20.

19. Zhou K, Mai H, Zheng S, Cai W, Yang X, Chen Z, Zhan B. OTUB1-mediated deubiquitination of FOXM1 up-regulates ECT-2 to promote tumor progression in renal cell carcinoma. Cell Biosci. 2020;10:50.

20. Liu J, Liu ZX, Wu QN, Lu YX, Wong CW, Miao L, Wang Y, Wang Z, Jin Y, He MM, Ren C, Wang DS, Chen DL, Pu HY, Feng L, Li B, Xie D, Zeng MS, Huang P, Lin A, Lin D, Xu RH, Ju HQ. Long noncoding RNA AGPG regulates PFKFB3-mediated tumor glycolytic reprogramming. Nat Commun. 2020;11(1):1507.

21. Lei Y, Chen T, Li Y, Shang M, Zhang Y, Jin Y, Yu Q, Guo F, Wang T. O-GlcNAcylation of PFKFB3 is required for tumor cell proliferation under hypoxia. Oncogenesis. 2020;9(2):21.

22. Ma H, Zhang J, Zhou L, Wen S, Tang HY, Jiang B, Zhang F, Suleman M, Sun D, Chen A, Zhao W, Lin F, Tsau MT, Shih LM, Xie C, Li X, Lin D, Hung LM, Cheng ML, Li Q. c-Src Promotes Tumorigenesis and 
Tumor Progression by Activating PFKFB3. Cell Rep. 2020;30(12):4235-49.

23. Zscharnack K, Kessler R, Bleichert F, Warnke JP, Eschrich K. The PFKFB3 splice variant UBI2K4 is downregulated in high-grade astrocytomas and impedes the growth of U87 glioblastoma cells. Neuropathol Appl Neurobiol. 2009;35(6):566-78.

24. Calvo MN, Bartrons R, Castaño E, Perales JC, Navarro-Sabaté A, Manzano A. PFKFB3 gene silencing decreases glycolysis, induces cell-cycle delay and inhibits anchorage-independent growth in HeLa cells. FEBS Lett. 2006;580(13):3308-14.

25. Møller MB. P27 in cell cycle control and cancer. Leuk Lymphoma. 2000;39(1-2):19-27.

26. Yalcin A, Clem BF, Simmons A, Lane A, Nelson K, Clem AL, Brock E, Siow D, Wattenberg B, Telang S, Chesney J. Nuclear targeting of 6-phosphofructo-2-kinase (PFKFB3) increases proliferation via cyclin-dependent kinases. J Biol Chem. 2009;284(36):24223-32.

\section{Figures}


a

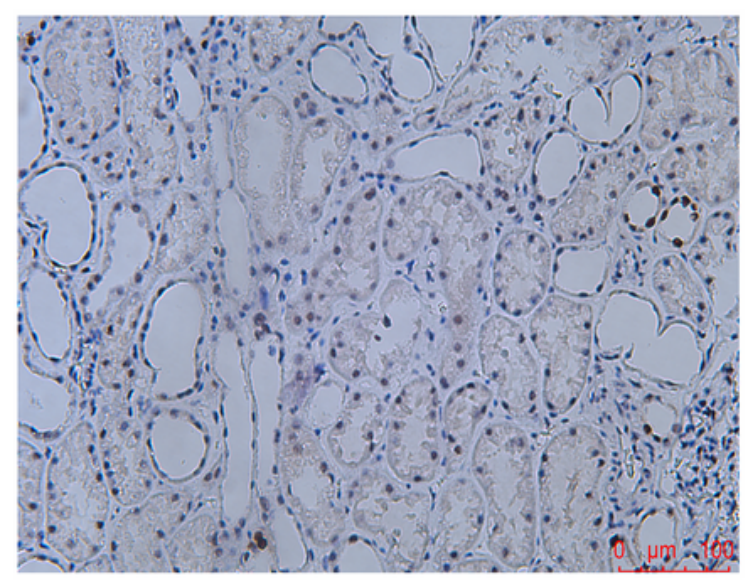

b

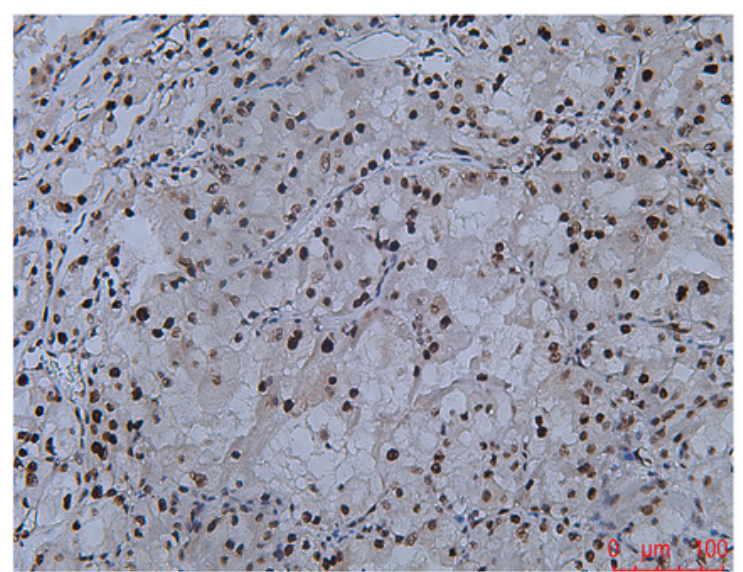

c

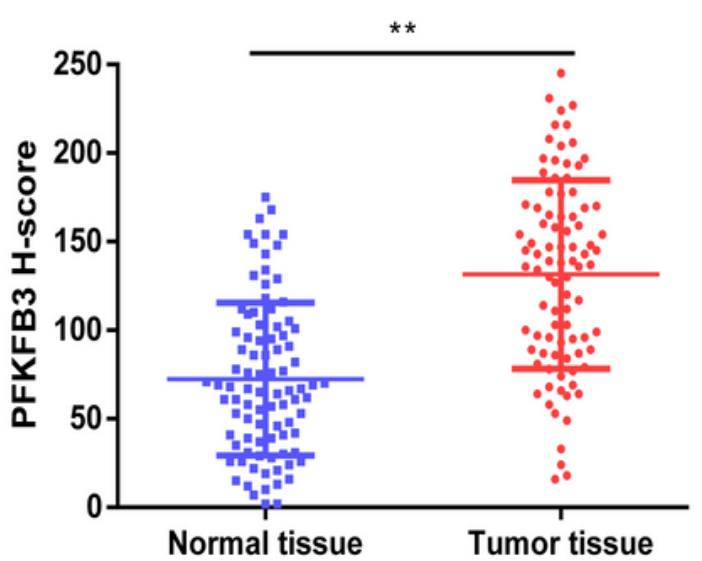

e

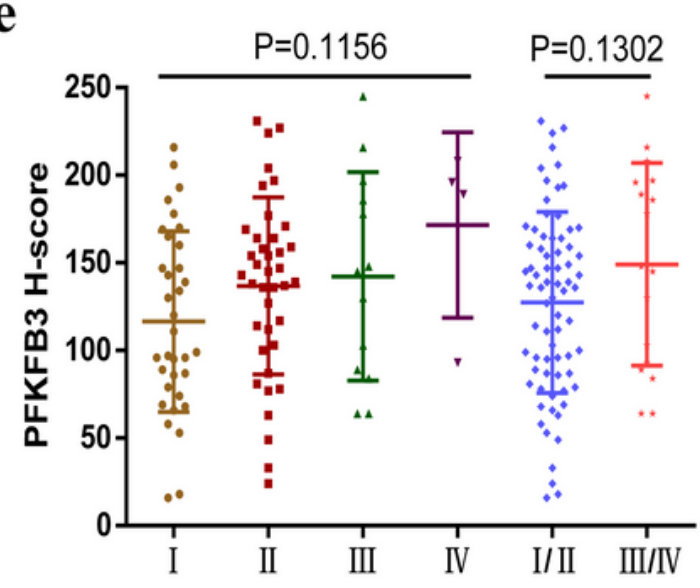

Furhman grade d

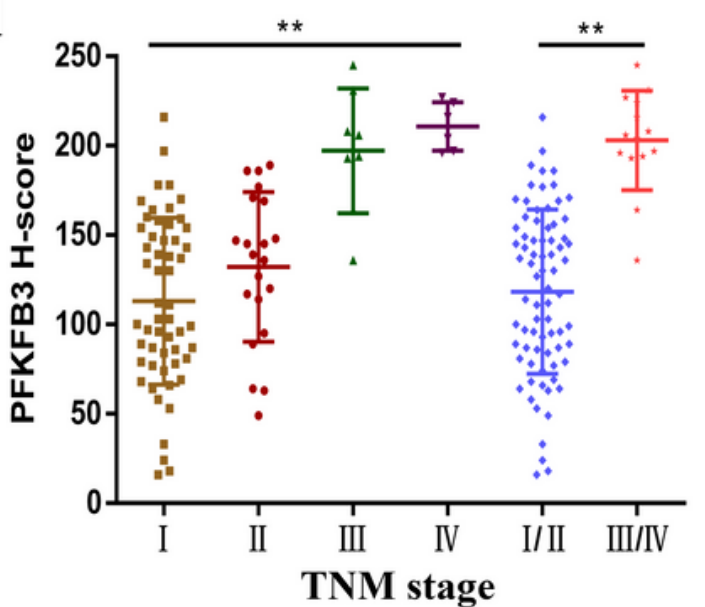

f

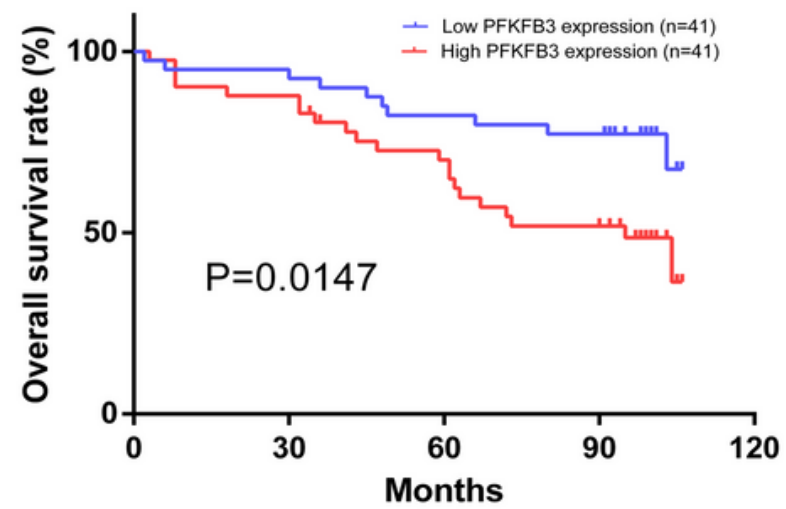

Figure 1

PFKFB3 protein is overexpressed in human ccRCC specimens and positively correlates with advanced TNM stage and poor prognosis of ccRCC patients. (a) Representative images of adjacent normal tissues with low expression of PFKFB3. Scale bar, $100 \mu \mathrm{m}$. (b) Representative images of ccRCC tissues with high expression of PFKFB3. Scale bar, $100 \mu \mathrm{m}$. (c) A plot of the H-score of PFKFB3 in ccRCC tissues and adjacent normal tissues. (ccRCC=131.52 \pm 5.61 vs. Normal=72.36 $\pm 4.55, \mathrm{P} \otimes 0.01)$. (d-e) H-score of PFKFB3 
in different stages (TMN) and grades (Fuhrman) of ccRCC patients. (f) Kaplan-Meier analysis of overall survival time in ccRCC cases based on PFKFB3 expression. The results are presented as the mean \pm SD. *, $\mathrm{P} \otimes 0.05 ; * \star, P \otimes 0.01$.

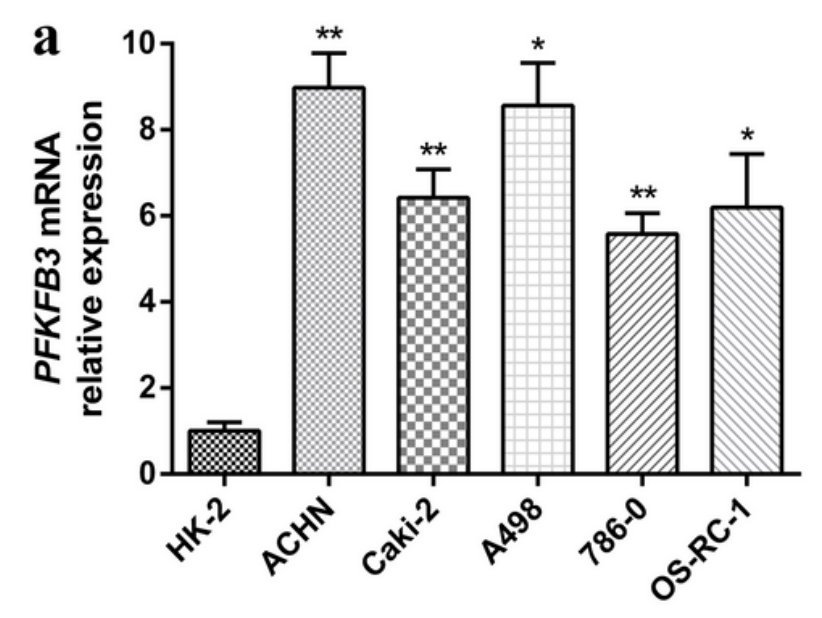

b
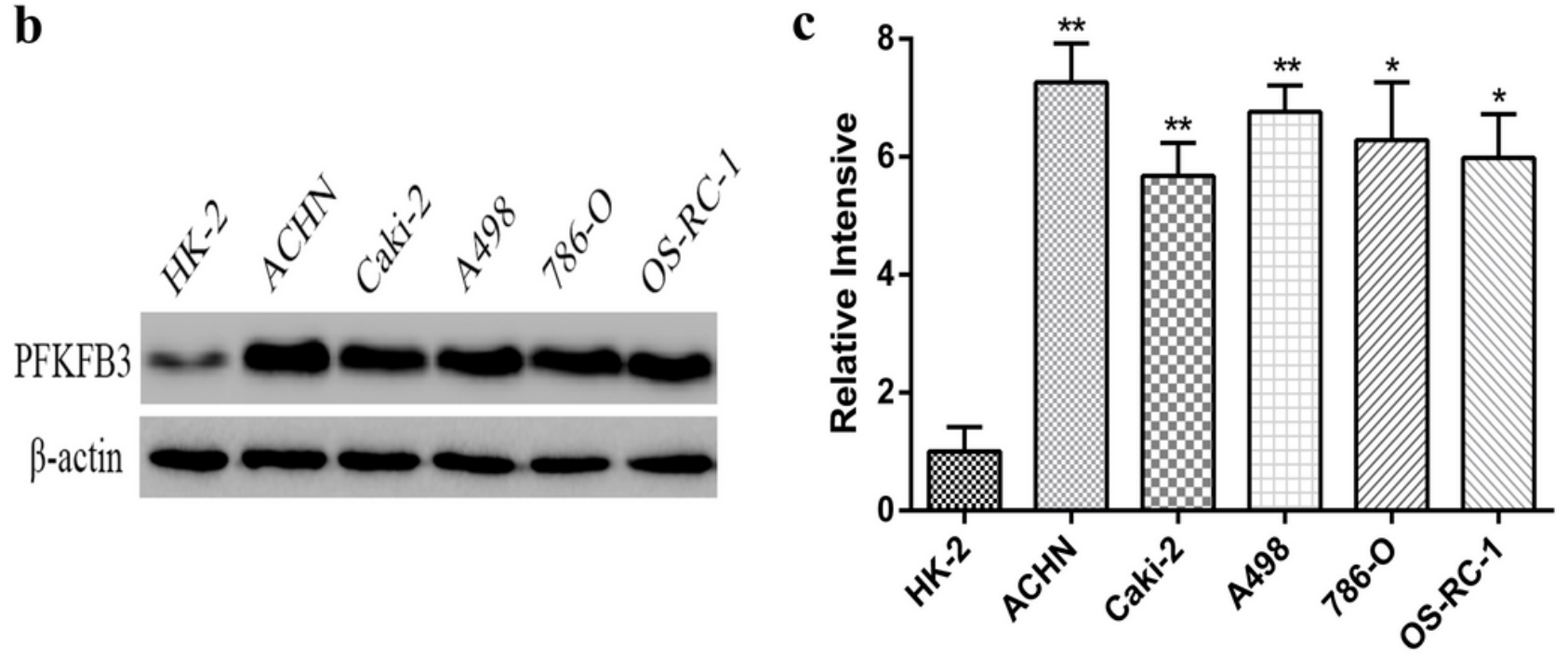

Figure 2

PFKFB3 expression levels are higher in RCC cells. (a) PFKFB3 mRNA levels in a panel of RCC cell lines (ACHN, Caki-2, A498, 786-O and OS-RC-1) and in normal human proximal tubule epithelial cell line (HK-2) were determined by qRT-PCR. $\beta$-actin was used as the intern control gene. (b-c) Protein levels of PFKFB3 in RCC cells were determined by Western blot analysis. $\beta$-actin was used as the intern control protein. The results are presented as the mean \pm SD. ${ }^{*}, \mathrm{P} \otimes 0.05 ; * \star, P \llbracket 0.01$. 
a

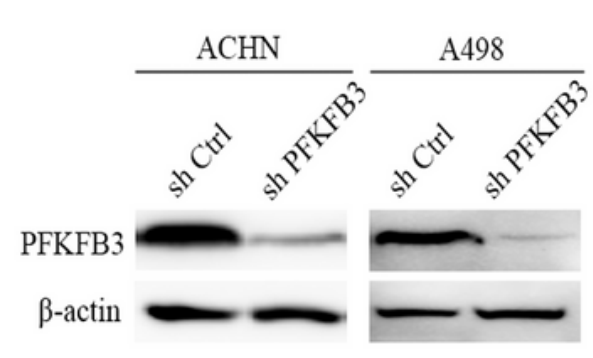

c

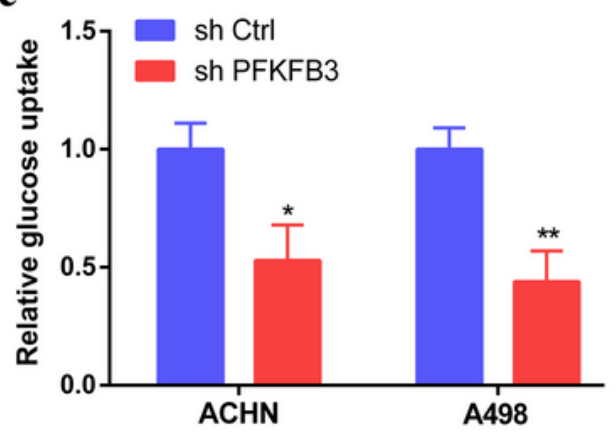

e

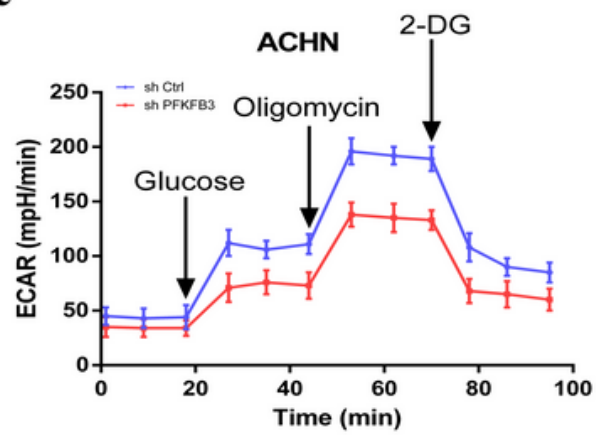

g

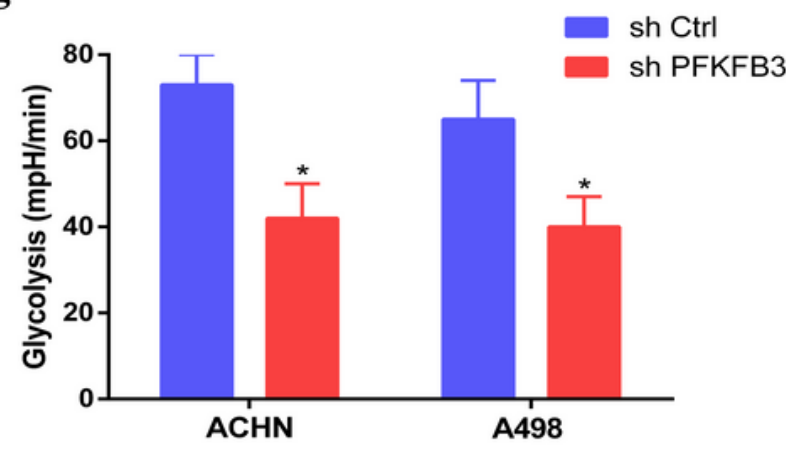

b

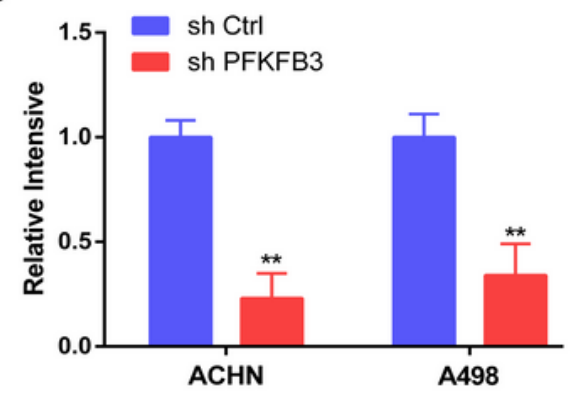

d

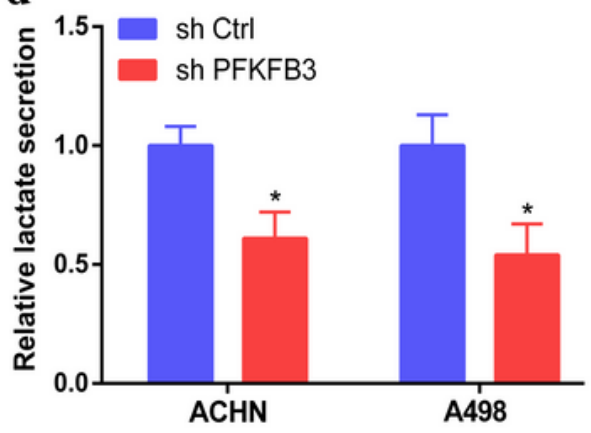

f

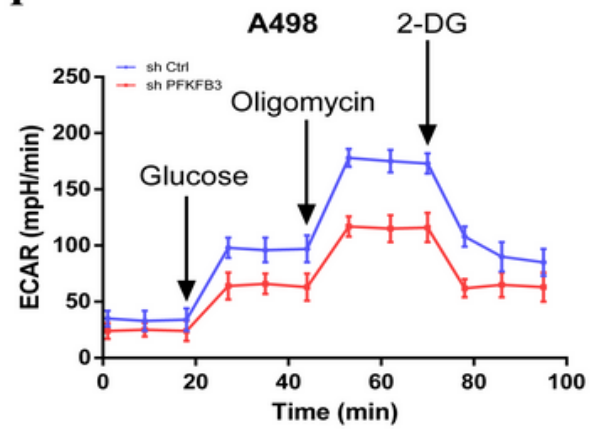

\section{Figure 3}

Knockdown of PFKFB3 inhibits glycolysis in RCC cells. (a-b) After shRNA transfection, Western blot analysis was used to confirm the knockdown of PFKFB3 in ACHN and A498 cells. (c-d) Measurement of relative glucose uptake and lactate secretion in transfected ACHN and A498 cells. (e-f) ECAR levels were detected in transfected ACHN and A498 cells using an XF Extracellular Flux Analyzer. (g) Glycolysis 
capacity was further quantified by ECAR analysis. The results are presented as the mean $\pm S D .{ }^{*}, P \otimes 0.05$; **, $\mathrm{P} \otimes 0.01$.

a

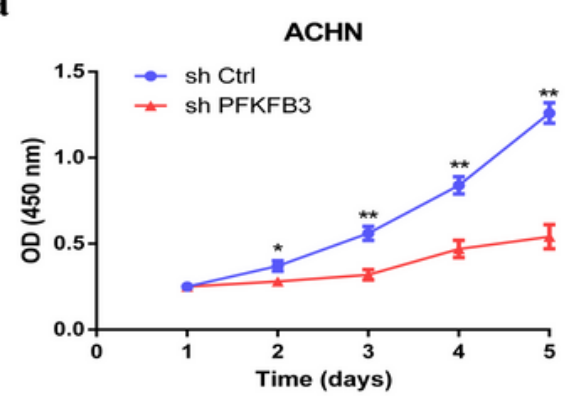

c

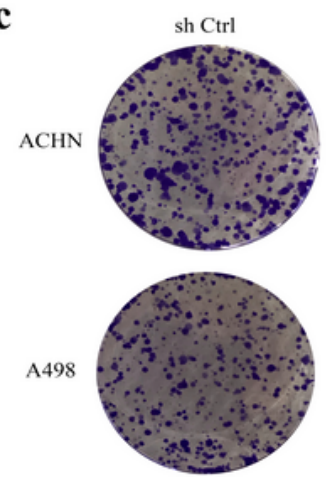

e

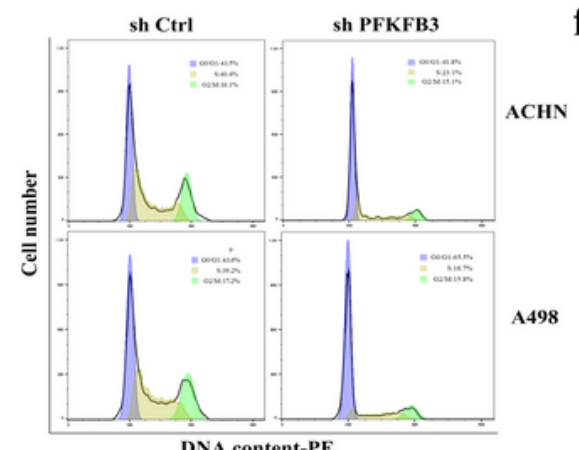

g

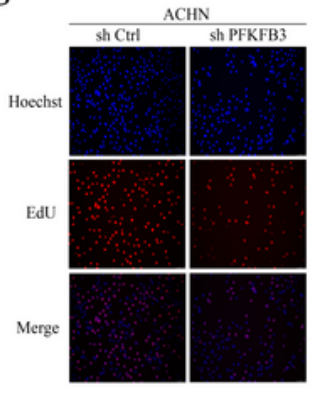

f

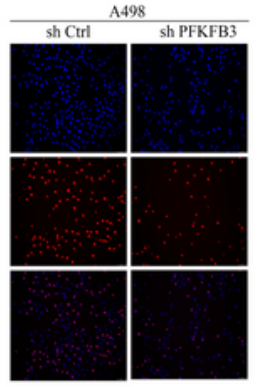

b

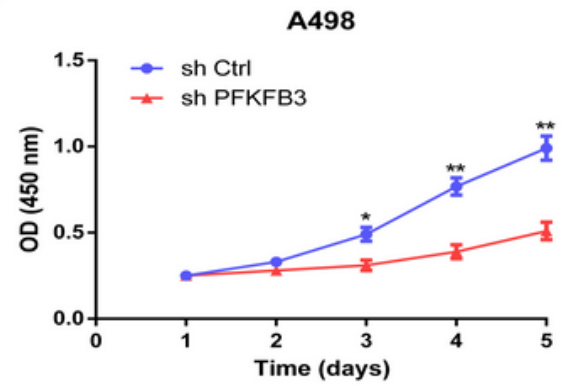

d

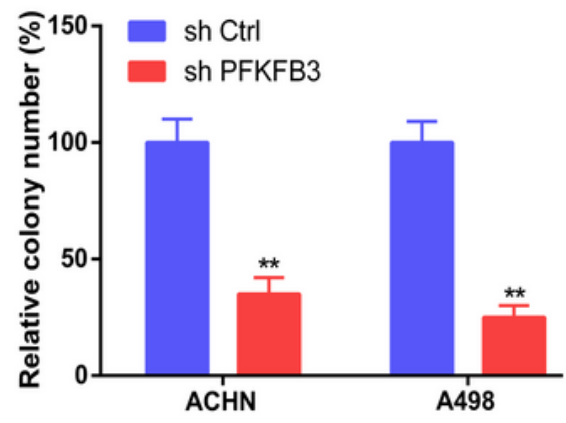

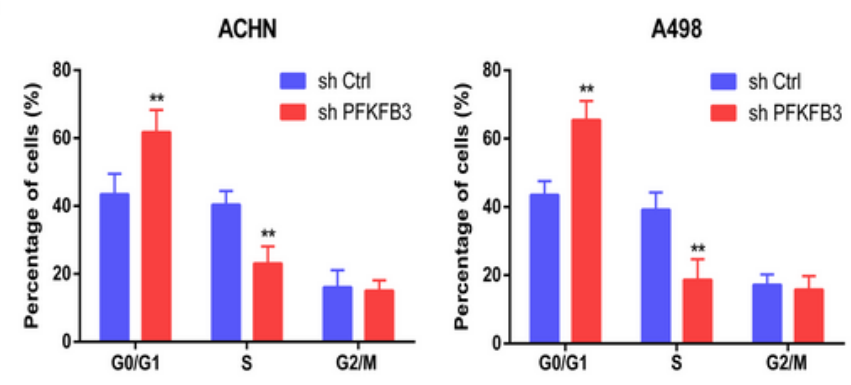

h

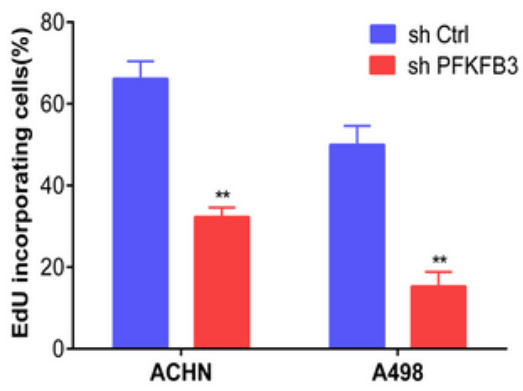

Figure 4

Downregulation of PFKFB3 suppresses proliferation and G1/S transition in RCC cells. (a) CCK-8 assays were used to determine the viability of the indicated cells. (b-c) Representative images and relative quantification of crystal violet-stained cell colonies analyzed by colony formation assay. (d) Flow 
cytometry assays were performed to analyze cell cycle distribution of the indicated cells. (e) Statistical analysis of the indicated cells (\%) in each cell cycle phase. (f-g) Representative micrographs and quantification of EdU-incorporated cells in the indicated cells. The results are presented as the mean $\pm S D$. $\star, P \otimes 0.05 ; * \star, P \otimes 0.01$.

a

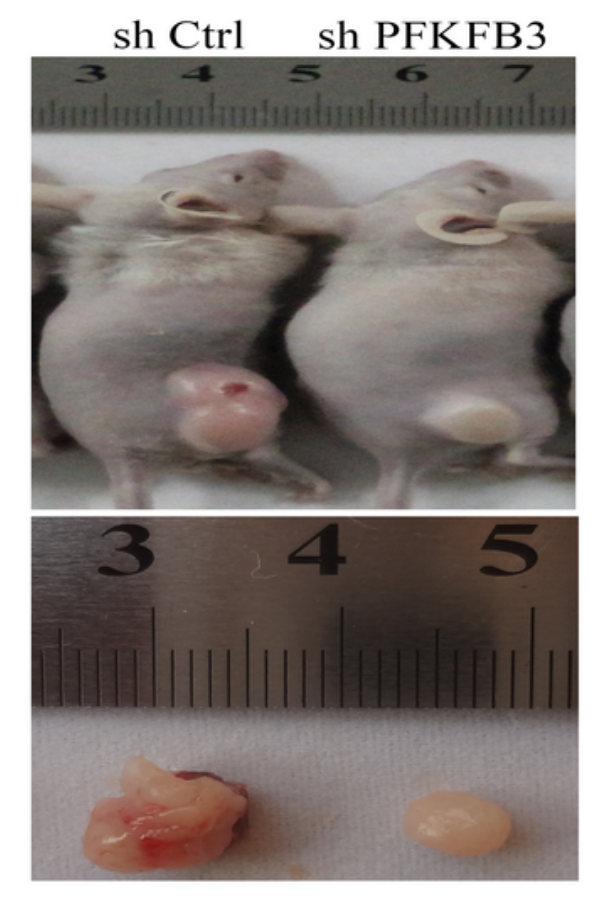

d

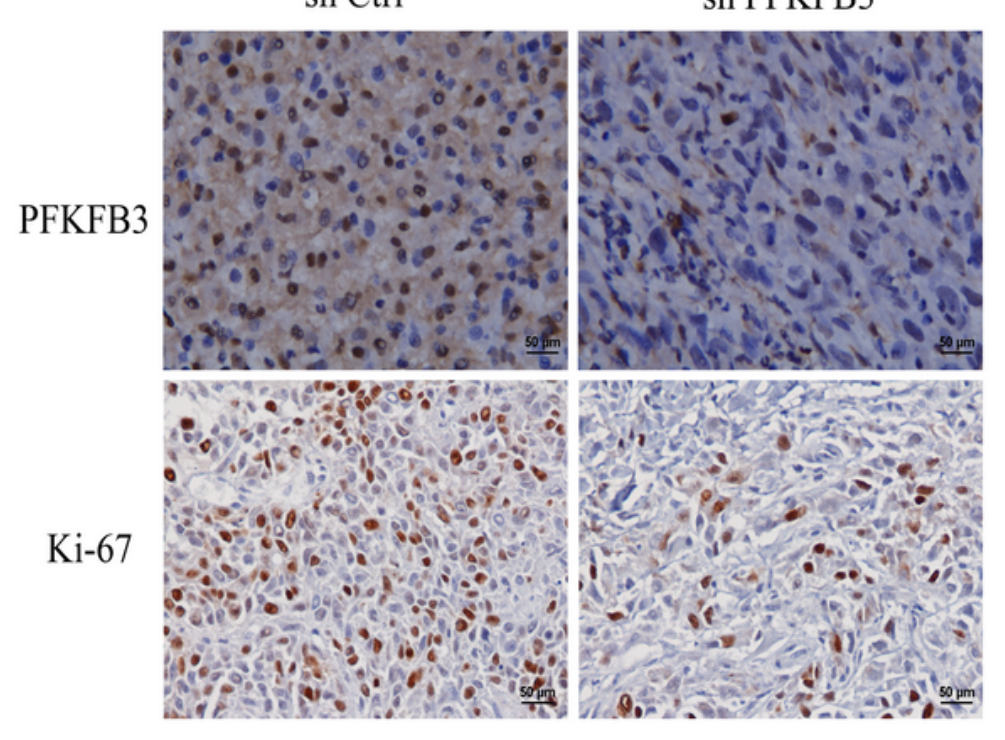

b

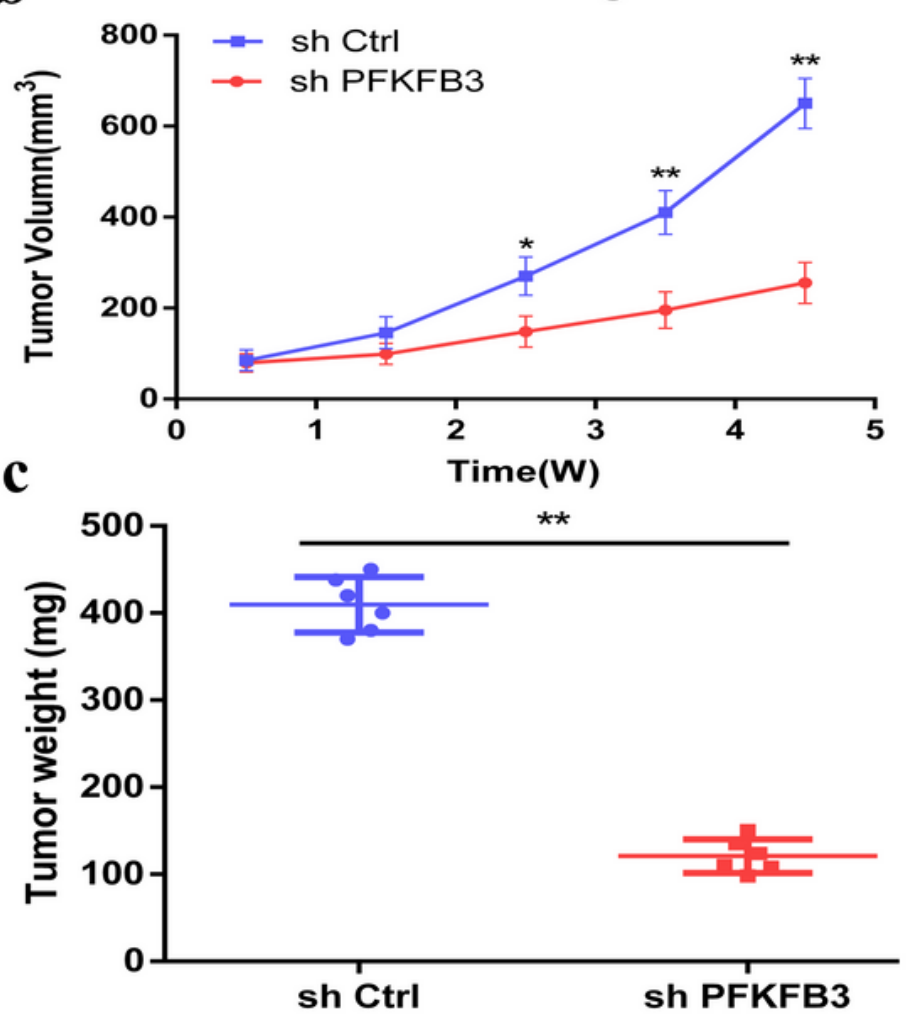

$\mathbf{e}$

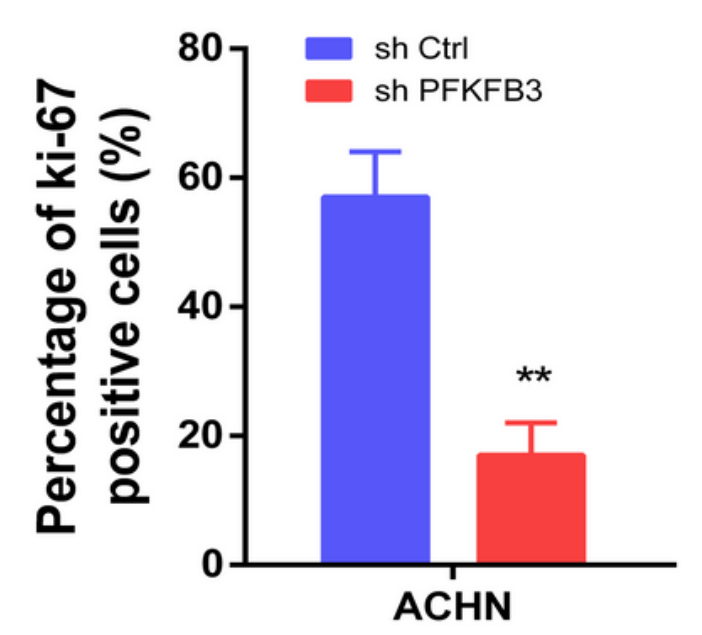

Figure 5 
PFKFB3 knockdown inhibits RCC tumor growth in vivo. (a) Representative images of ACHN cell-based tumor formation in mice under different conditions. (b) Statistical analysis of ACHN cell-based tumor volumes in nude mice. (c) Statistical analysis of $\mathrm{ACHN}$ cell-based tumor weight in nude mice. (d) Representative IHC images of PFKFB3 and Ki-67 in randomly selected ACHN cell-based tumors from each group. Scale bar, $50 \mu \mathrm{m}$. (e) Quantification of IHC staining of Ki-67 in ACHN cell-based tumors. The results are presented as the mean $\pm S D .{ }^{*}, \mathrm{P} \otimes 0.05 ; * \star, P \otimes 0.01$. 